Aus der dermatologischen Universitätsklinik des Prof. Dr. Kaposi in Wien.

\title{
Ueber die Trichorrhexis nodosa barbae (Kaposi) und ihren Erreger.
}

\author{
Von
}

Docenten Phil. u. Med. Dr. Eduard Spiegler,

Assistent der Klinik.

(Hierzu Taf. V.)

Die unter diesem Namen bekannte Krankheit der Haare charakterisirt sich klinisch bekanntlich durch folgende Momente:

Die Haare erscheinen an ihrem freien Ende und am Schafte mit einem oder mehreren weissen Pünktchen besetzt, und erregen so den Anschein, als sässen Nisse daran. Andere Haare erscheinen wie abgerissen oder wie abgebrannt, oder fasern sich an ihren Enden besenförmig auf. Fasst man ein solches Haar an seinem Ende, um es auszuziehen, so geschieht es regelmässig, dass dasselbe an der einem solchen Knötchen entsprechenden Stelle abreisst. Bei dem Versuche ein solches Haar zu biegen, knickt es an der betreffenden Stelle immer ein.

Da dieses Abreissen an den unmittelbar afficirten Stellen durch mechanische Einwirkungen, als Kämmen, Waschen, Bürsten immer wieder verursacht wird und sich am Haarschaft immer wieder frische Knötchen bilden, an denen sich das alte Spiel wiederholt, ist es begreiflich, dass die Haare auf die Weise immer kürzer werden.

In unseren Gegenden wird diese Affection in den Barthaaren der Männer sehr häufig beobachtet, in den Kopfhaaren der Frauen selten, in den Kopfhaaren der Männer kaum, wenigstens erinnere ich mich nicht einen diesbezïglichen Fall gesehen zu haben. 
Namentlich fand ich diese Affection sehr häufig in den Barthaaren bei Aerzten, ohne dass ich indess im Stande wäre, dies mit bestimmten Verhältnissen in Verbindung zu bringen, vielleicht nur, weil Aerzte mehr darauf aufmerksam werden und mehr Gelegenheit finden, sich an Fachcollegen desshalb zu wenden.

\section{Historisches.}

Die Trichorrhexis nodosa fand ihren ersten Beschreiber in Beigel, dessen Publication über "Auftreibung und Berstendes Haares" in den Denkschriften der Wiener kaiserlichen Alzademie der Wissenschaften mitgetheilt ist. Beigel suchte die Ursache dieser Veränderung auf Gasentwickelung im Inneren des Haares zurückzuführen.

Der nächste Autor, der sich mit dieser Veränderung der Haare beschäftigte, ist Samuel Wilks, der in seinen Lectures of pathological Anatomy 1857 dieses Uebel kurz erwähnt. Als Ursache desselben spricht er eine durch Pilze hervorgerufene Nutritionsstörung an, während wieder Frasmus Wilson sich hinsichtlich der Aetiologie Beigel anschliesst. Devergie berichtete im Jahre 1871 und bald darauf Luigi Billi über ein ähnliches Uebel und schlug mit Rücksicht auf die federnähnliche Zerfaserung der Haare den Namen Trichoptilosis vor. Doch weder der von Wilson vorgeschlagene Name $\mathrm{Tr}$ choclasis s. Trichoclasia, noch auch der viel spätere von Duhring - fragilitas crinium, welcher lediglich ein Symptom dieser Affection betrifft, fand Eingang, vielmehr wird dieselbe seit Kaposi's Vorschlag 1870 allgemein als Trichor rexis nodos a bezeichnet.

Nun machte Desenne im Jahre 1878 in der Pariser Akademie der Wissenschaften Vittheilung über eine eigenthümliche Haarkrankheit, die an den Eingeborenen von Columbia in der Provinz Cauca beobachtet wird, und die sich durch Bildung steinharter Knötchen, namentlich an den Enden der Barthaare manifestirt und die im Spanischen "Piedra" genannt wird. Diese Veränderung, namentlich aber die Härte der Knötchen, soll auf Anlagerung von Epithel-Massen um die Haare beruhen. Diese Zellen sind regelmässig angeordnet, von polygonaler Form und von 12-15 $\mu$ Durchmesser. Die Knötchen selbst sind so hart, dass beim Durchziehen eines Kammes ein Crepitation ähnliches Geräusch entsteht und diese Knötchen selbst der Schneide des Scalpells widerstehen - daher der Name "Piedra“ (Stein). Die Frage, ob es sich hiebei um eine parasitäre Krankheit handelt, lässt Desenne im Gegensatze zu Osorio, dem ersten Beobachter dieser Affection, unentschieden.

Trotz dieser so auffalligen Verschiedenheit dieser Affection von Trichorrhexis nodos a hat sich namentlich in England an Desen ne's Publication eine lebhafte Controverse darüber entwickelt, ob diese Affectionen nicht identisch wären. 
Malley hebt die Aehnlichkeit der in England beobachteten Fälle, die sich offenbar auf Trichorrhexis nodosa beziehen, mit Piedra hervor, und macht auf den Umstand aufmerksam, dass seine Fälle jeder Behandlung trotzten.

Cheadle hat eine der Piedra ähnliche Veränderung der Haare schon vorher beobachtet und unterscheidet sie von Trichorrhexis nodosa. Er fand in den kranken Haaren Sporen. Ueber solche berichtet auch Malcolm Morris und Hoggan, während S. Wilks keine solchen fand, doch lässt MaIcolm Morris die Identität mit Trichorrhexis dahingestellt. In einer släteren gemeinsamen Publication von M. Morris und $\mathrm{Cheadle}$ trennen diese Autoren die Piedra von Trichorrhexis nodosa und nennen die von ihnen beschriebene, mit letzterer wohl identische Affection, Tinea nodosa.

Whitla bält die Veränderung überhaupt nicht für pathologisch, während Roeser sie mit der Trichoptilosis Devergie and der Scissura pilorum der alten Autoren identificirt und in einer etwas unklaren Darstellung sie für den Beginn einer zur Kahlheit führenden Erkrankung erklärt.

M.c. C. Anderson berichtet über einen Fall von hereditärer Trichorrhexis ${ }^{1}$ ) mit genauester Wiedergabe des entsprechenden Familienstammbaumes.

Von deutschen Autoren, welche die Krankheit sämmtlich als Trichorrhexis nodosa anführen, bezieht Wolfsberg deren Entstehung auf mechanische Misshandlung der Haare, Eich horst auf einen Degenerationsvorgang im Sinne der Verfettung, Vir chow, Schwimmer, S. Kohn auf Ernährungsstörung. Kaposi und Michelson stellen diese Affection in eine Linie mit der Spitzenzersplitterung und sehen sie als Folge des Austrocknens bei geschwächter Ernährung und verminderter Säftezufuhr an, wobei indess Kaposi selbst auf das Unzulängliche dieser Erklärung, weshalb die Haare an einzelnen Stellen sich auftreiben und abbrechen, hinweist.

Das dieser Veränderung entsprechende mikroskopische Bild war mit trefflichen Abbildungen versehen, schon so oft Gegenstand der Beschreibung, dass ich auf die nähere Schilderung der diesbezüglichen Verhältnisse nicht eingehe, umsomehr, da sich meine Befunde mit denen der friheren Autoren decken. Hingegen möchte ich in dieser Richtung auf einen bisher nicht hervorgehobenen Umstand aufmerksam machen: wenn man nämlich die Trichorrhexis-Haare färbt, so behalten die afficirten den Knötchen entsprechenden Stellen mit

1) Es handelt sich hier selbstverständlich um Uebertragung durch gemeinsames Kammzeug von Individuum auf Individuum in der Familie, wie aus den folgenden Erörterungen hervorgehen wird. 
der grössten Hartnäckigkeit den Farbstoff zurück. Aber nicht nur diese Stellen verhalten sich so, sondern auch andere scheinbar vollkommen normale und unveränderte. Frst bei genauerer Betrachtung nimmt man wahr, dass das Haar auch an solchen Stellen um ein geringfügiges aufgetrieben und verbreitert erscheint. Es handelt sich hier wohl um das Anfangsstadium jener zur Auffaserung und Zerklüftung führenden Veränderung, die schon so frühzeitig durch ihr verschiedenes Verhalten gegen Farbstoffe die tieferen Veränderungen in diesem Theile documentirt.

Trichorrhexis nodosa des Kopfhares, wie sie $\mathrm{Ho}_{0}$ dara bei den Konstantinopeler Frauen beobachtet hat, kommt, wie schon vor erwähnt, bei den Frauen in unseren Gegenden nur selten ror, sehr häufig hingegen bei uns eine klinisch, wie es scheint, identische Affection der Barthaare bei Männern. Diese hinwieder gehört. wie Hodara mündlich mir mitzutheilen die Güte hatte, in Konstantinopel zu den Seltenheiten. Es schien nun interessant, zu untersuchen, in wie weit diese beiden Affectionen pathologisch und aetiologisch zusammengehörig seien.

In verhältnissmässig kurzer Zeit ist es mir gelungen, diese Affection in den Barthaaren von zehn Collegen zu finden, die so gütig waren, mir kranke Haare zur Untersuchung zur Verfügung zu stellen. Sämmtliche standen im Alter um 30 Jahre und waren schon seit langem mit diesem Uebel behaftet. Obwohl ich auch in unserem klinischen Ambulatorium die Aufmerksamkeit hierauf richtete, fand ich unter den tausenden Patienten nur sebr wenige Fälle von Trichorrhexis barbae. Seither sind mir noch mehr Fälle von Trichorrhexis barbae bei Aerzten begegnet.

Eine sehr befriedigende Aufklärung über die Ursache dieser Affection brachte vor drei Jahren Hodara, welcher Trichorrhexis-Kopfhaare von Konstantinopeler Frauen zum Untersuchungsobjecte hatte. Hodara fand in den so veränderten Haaren regelmässig bestimmte Mikroorganismen, welche er nicht nur mikroskopisch nachwies, sondern auch züchtete. Mit Hilfe der Culturen gelang es ihm, die gleiche Affection auch an gesunden Haaren zu erzeugen und so den unanfecht- 
baren Beweis zu erbringen, dass jene Mikroorganismen wirklich die Erreger dieser Krankheit sind.

\section{Nachweis der Mikroorganismen in den Haaren.}

Zum Zwecke des Nachweises der Mikroorganismen in den Haaren bin ich anfangs genau so vorgegangen wie Hodara. Die Haare wurden zunächst in Aether oder in Alkohol-Aether gebracht, um dieselben zu entfetten. Hierauf wurden sie in die gewöhnliche käufliche Lösung von Wasserstoffhyperoxyd gebracht, und in dieser so lange belassen, bis sie vollständig entfärbt waren, was je nach ihrer Pigmentirung verschieden lange Zeit in Anspruch nahm. Nach Auswaschen in sterilem Wasser wurden die Haare in eine frisch bereitete Lösung von Anilinwasser-Gentianaviolett für 2-4 Minuten gebracht, in Wasser ausgewaschen und in Lugol's Lösung fixirt, oder für 2 Minuten in eine Lösung von Wasserstoffsuperoxyd, zu der man einige Jodkalikrystalle beifügt, wodurch Jod frei wird, gebracht. Abermaliges Auswaschen in Wasser, Trocknen mit Filtrirpapier, hierauf Einlegen in Anilinöl. Dieses wiederholt wechseln und zwar so lange, bis das Haar, welches den Farbstoff sehr begierig aufgenommen hat und sehr zähe festhält, nahezu vollkommen entfärbt ist. Dies dauert oft mehrere Tage und es zeigt sich hiebei, dass die den Knoten entsprechenden Stellen den Farbstoff besonders hartnäckig zurückhalten. Hierauf wird das Haar in Xylol oder Bergamottöl gebracht und in Canadabalsam eingeschlossen. Nach meinen Beobachtungen hat es sich aber als viel zweckmässiger erwiesen, wenn man die Haare in der Gentiana-Anilinwasserlösung nur ganz kurze Zeit - 20 bis 30 Secunden - färbt, im übrigen aber in der vorbeschriebenen Weise vorgeht. Diese kurze Färbezeit reicht nämlich zur Darstellung der Mikroorganismen vollkommen aus, wobei man noch den Vortheil hat, dass man nicht so lange mit Anilinöl zu differenciren braucht, bei welcher Procedur es sehr leicht geschehen kann, dass sich die Mikroorganismen selbst gleichfalls ganz entfärben.

In allen so vorbereiteten Haaren findet man nur äusserst vereinzelte Stäbchen, sehr reichlich aber Coccen ähnliche Gebilde, und zwar namentlich in den aufgefaserten, mikroskopisch 
den weissen Punkten entsprechenden Partien. Diese Gebilde findet man in abnehmender Menge noch oberhalb und unterhalb des aufgefaserten Theiles des Haares. Bei einzelnen Haaren, welche nahe der Haut einen Knoten tragen, findet man diese Formen auch im subepidermoidalen Theile des Haares. Aber auch in einzelnen Zellen der Follikelwand, welche beim Ausziehen von Haaren mit diesen mitgerissen werden und im mechanischen Zusammenhange mit denselben geblieben sind, habe ich in einzelnen Präparaten diese Coccen ähnlichen Bilder gesehen. Näherer Aufschluss über die Verbreitung der Mikroorganismen in dem perifolliculären Gewebe könnte nur durch die histologische Untersuchung eines entsprechenden Hautstückes erfolgen, wozu sich mir bis nun Gelegenheit noch nicht geboten hat.

Bei Betrachtung dieser Bilder ist es allerdings nicht leicht, sich die Ueberzeugung zu verschaffen, dass die vorliegenden Gebilde Mikroorganismen entsprechen. Die Culturen jedoch, von denen später ausführlicher die Rede sein wird, beweisen dies zur Genïge. Man findet übrigens in den Haaren selbst deutliche Stäbchen, die sich mit denjenigen, die durch Züchtung aus Trichorrhexis-Haaren gewonnen waren, vollkommen übereinstimmen. Wenn man nach der erwähnten Methode präparirte Trichorrhexis-Haare näher untersucht, so findet man mitunter neben den beschriebenen Gebilden Stäbchen von verschiedener Länge und zwar ron $1-12 \mu$ und ausserdem $\mathrm{Sch}$ einfäden, in denen sich die einzelnen Glieder deutlich wahrnehmen lassen. Diese Bilder sind, wie aus der Abbildung hervorgeht, so überzeugend, dass in Bezug auf ihre Uebereinstimmung mit den aus Culturen gewonnenen gar kein Zweifel obwalten kann. Diese Bilder wurden $b$ ishe $r$ nicht beobachtet, sonst würden weniger Zweifel über die parasitäre Natur der Trichorrhexis in der hier darzulegenden Richtung aufgetaucht sein. Allerdings muss ich Hodara zustimmen, dass erst die Cultur die richtige Deutung seiner Bilder ermöglicht.

Die vorerwähnten Mittheilungen älterer Autoren über Mikroorganismen in Trichorrhexis-Haaren, sind insoferne zweifelhaft, als dieselben in ungebleichten und ungefärbten Haaren 
nicht klar sind und leicht mit Pigmentkörnchen verwechselt werden können.

Uebrigens gestatten auch andere Färbemethoden den Nachweis der Mikruorganismen; so z. B. kann man die Haare mit gewöbnlichem Carbolfuchsin färben und in Anilin entfärben, doch habe ich mich mit Vorliebe der erwähnten Methoden bedient.

\section{Züchtung der Mikroorganismen.}

Auch hier wählte ich dieselbe Methode, deren sich $\mathrm{Ho}$ dara bedieste.

Zur Abtödtung oberflächlich sitzender Mikroorganismen wurden die Haare nach Entfettung in Aether für einige Tage in absoluten Alkohol gebracht, auf Agarplatten übertragen und diese in den Brutofen gebracht. Bereits nach 24 Stunden war manchmal längs eines Theiles des Haarschaftes, manchmal aber längs des ganzen Haares, den subepidermoidalen Theil desselben mit inbegriffeu, ein weisslich grauer Saum zu sehen, der circa $2 \mathrm{Mm}$. breit war. Nach weiteren 24 Stunden wurde derselbe um ein geringes breiter und blieb dann sowohl der Länge als der Breite nach stationär. Bei schwacher Vergrösserung bot diese Cultur insoferne gar nichts charakteristisches, als nur eine feinzackige und feinkörnige durchscheinende Masse sichtbar war. Diese Art und Weise des Wachsthumes der Mikroorganismen auf der Agarplatte gibt einen Fingerzeig dafür, wie dieselben im Haare selbst verbreitet sind. Demnach ist die Ausbreitung der Bakterien im Haare manchmal auf eine Theilstrecke desselben beschränkt, manchmal sind sie in einem grösseren Theile des Haares verbreitet, manchmal wiederum erstrecken sie sich bis in den subepidermoidalen Theil hinein. Wir kommen übrigens auf dieses rom Standpunkt der Therapie wichtige Verhalten weiter unten zurück.

Impfstriche von solchen Colonien auf schief gelegtes Agar geben bereits nach 24 Stunden zahlreiche kleine, runde zart weisslichgraue Colonien, die nach weiteren 24 Stunden an Ausbreitung zunehmen und confluiren, dann aber stationär bleiben. 
Ein sehr charakteristisches Aussehen hat die Stichcultur a uf Agar. Um die Einstichstelle bildet sich an der Oberfläche des Agars schon nach 24 Stunden ein schmaler Saum, der nach einigen Tagen die Oberfläche des Agars ganz bedeckt und sich zu einem weissschimmernden Ueberzug umbildet, der bei weiterem Wachsthum ungefähr die Dicke von $1 \mathrm{Mm}$. erreicht. Der Stichcanal selbst erinnert vielfach an Stichculturen von Anthrax. Die Colonien nehmen in ihrem Wachsthum um den Stichcanal herum ungeführ die Form einer Fichte an, indem radial von diesem ein Strahlensystem von kurzen Zweigen auswächst; dem Umstande entsprechend, dass an der Einstichstelle eine grössere Menge von Keimen deponirt wird, als in den tieferen Schichten, ist die Entwicklung daselbst entsprechend reichlicher. Im Laufe des weiteren Wachsthums wird die fichtenähnliche Configuration der Cultur insoferne verändert, als die einzelnen Arme zusammenfliessen und so scheibenförmige Gebilde, welche von oben nach unten, entsprechend dem ursprünglichen Verhalten der Strahlen selbst, an Grösse abnehmen.

Auf Kartoffeln gedeihen diese Bakterien bei Brutofentemperatur ausserordentlich üppig, indem schon nach 30 Stunden ein glänzender Belag von rahmiger Consistenz sichtbar wird, welcher nach 48 Stunden noch zunimmt, dann aber stationär bleibt.

Stichculturen a uf Gelatine verhalten sich folgendermassen: Bereits nach 24 Stunden bemerkt man bei Lupenvergrösserung ein System von ganz kurzen, äusserst zarten Armen, welche ausserordentlich enge aneinander angeordnet auf den Sticheanal senkrecht stehen und um diesen ein Strahlensystem bilden. Nach einigen Tagen vergrössern sich diese Strahlen nur unwesentlich, wogegen sich aber an der Einstichstelle ein Knöpfchen von graulich weisser Farbe bildet, welches, unter die Oberfläche der Gelatine einsinkt - das erste Zeichen beginnender langsamer Verflüssigung - und sich des weiteren noch vergrössert. Nach circa 10 Tagen beginnt die Gelatine daselbst flüssig zu werden und im Laufe von einigen Wochen erscheint der ganze Inhalt des Rölirchens verflüssigt.

Die auf den genannten Nährböden geziichteten Mikro- 
organismen sind Stäbchen, die in ihrer Grösse beträchtlichen Schwankungen unterliegen. So findet man Stäbchen, deren Länge von 1 bis $10 \mu$ variirt und deren Breite circa $1 / 2 \mu$ beträgt, ein Umstand, den ich besonders hervorheben muss, da sich in dieser Hinsicht manche Abweichungen ergeben zwischen den bisherigen Beobachtungen und den meinigen.

Hodar a berichtet nämlich, dass er Scheinfäden gefunden hätte, deren Länge „zwei, drei und mehr $\mu$ " beträgt. Ich habe zwar solche Fäden ebenfalls gesehen, aber ausserdem noch Fäden, deren Länge bis zu $10 \mu$ betrug, die sich aber auch bei genauester Untersuchung absolut nicht in einzelne Glieder haben auflösen lassen, sondern vielmehr sich als Einzelindividuen erwiesen. Es liegt nun nahe, anzunehmen, dass auch Hodara solche Stäbchen gesehen hat, dieselben aber für Scheinfäden gehalten haben dürte. Aber nicht nur in der selben Cultur kommen solche Schwankungen in der Grösse der Einzelindividuen vor, sondern dieselbe ist auch abhängig von dem Alter der Cultur. Während in ganz frischen Agarculturen die Grösse der einzelnen Stäbchen durchschnittlich $4 \mu$ beträgt, sind Stäbchen von $10 \mu$ in Culturen, die einige Monate alt sind, durchaus nichts ungewöhnliches - ein weiteres Symptom für den ausserordentlichen Polymorphismus dieser Bacterien, ja dieselben können, wie wir gezeigt haben. in Haaren, die gleichsam ihren natürlichen Nährboden bilden, bis zu $12 \mu$ lang werden.

Die Stäbchen erscheinen an den Enden leicht abgerundet und zeigen im hängenden Tropfen eine ungemein lebhafte Eigenbewegung. Sie färben sich sehr leicht auch mit dünneren wässerigen Lösungen von Anilinfarben, so mit Fuchsin oder Methylenblau. Hie und da kann durch Anlagerung eines oder mehrerer Stäbchen seitliche Aussprossung vorgetäuscht werden. Jedes einzelne dieser Stäbchen ist umgeben von einem schmalen hellen Hofe, welcher namentlich bei älteren Culturen und bei etwas längerer Einwirkung der Färbeflüssigkeit - es genügt sonst die Einwirkung einer wässrigen Fuchsinlösung durch einige Secunden - sich schwach rosa färbt. Methylenblau wird von diesem Halo nicht aufgenommen. Ausserdem sieht man schon an 24 Stunden alten Culturen innerhalb dieses 
Halo, die Stäbchen durch $1-3$ und mitunter mehr ungefärbte Stelleu unterbrochen sowie freie Körperchen, die mit solchen Segmenten an Form und Grösse übereinstimmen, und die gleichfalls bereits halonirt erscheinen. An Präparaten aus älteren Culturen beobachtet man, dass die Stäbchen die Neigung haben, grössere formlose Haufen zu bilden, welche ron einem den randständigen Individuen angehörendem gemeinsamen Halo umgeben sind. Diese früher erwähnte Segmentation ist offen. bar als Ansehickung zur Vermehrung durch Theilung der Länge nach zu deuten und die einzelnen Coccen ähnlichen Gebilde und kurzen Stäbchen als Producte dieses Vorganges, sowie als Uebergangsformen zu der Stäbchenform zu betrachten. Diese Bakterienart bildet ein ganz besonders schönes Studienobject für die erwähnte Vermehrungsart, welche die Ursache für die scheinbare Vielgestaltigkeit dieses Bacillus abgibt und hiebei die mannigfaltigsten Bilder liefert, wie sie auch Hodara beschrieben hat.

Von anderen Mikroorganismen gingen mir wiederholt Staphylococcen auf, ausserdem aber einmal ein Mikroorganismus, der auf Agar unter Bildung eines orangeähnlichen Farbstoffes sehr gut gedeiht und morphologisch dem TrichorrhexisBacillus ähnlich ist. Wie ich gleich vorausschicken will, handelt es sich lediglich um einen zufälligen Befund, der ebensowenig wie der des Staphylococcus in ätiologischer Hinsicht mit der Trichorrhexis in Zusammenhang steht, da Infectionsversuche mit dem chromogenen Mikroorganismus negativ ausfielen.

Es erübrigte also, um die Beweiskette zu schliessen, dass dieser Bacillus wirklich der Erreger der Trichorrhexis nodosa ist, nur noch, mittelst des aus den Barthaaren gezüchteten Bacillus, durch Uebertragung auf solche Individuen, welche von dieser Affection frei sind, Trichorrhexis nodosa $z u$ erzeugen. $\mathrm{Zu}$ diesem Zwecke nabm ich eine ältere Gelatincultur und trug sie mittelst eines sterilisirten Pinsels im Einverständniss des betreffenden Patienten, eines alten Mannes, auf seine Barthaare leicht auf. Trotz täglicher sorgfältiger Untersuchung konnte ich erst am 8. Tage nach der Impfung die charakteristischen Veränderungen an den Haaren wahr- 
nehmen, die sich auch mikroskopisch mit den an den Trichorhexis-Haaren beobachteten Bildern deckten.

Die Culturen, die ich aus solchen Haaren nach obiger Methode gewann, waren nicht zu unterscheiden von solchen, wie sie ausden TrichorrhexisHaaren dargestellt waren, die den erwähnten Fällen angehörten.

Es ist somit bewiesen, dass der e r wähnte Bacillus wirklich der Erreger dieser Krankheit ist. Auf die Behauptung, dass auch andere Mikroorganismen diese Krankheit erzeugen können, wäre zu erwiedern, dass hiefür der experimentelle Beweis durch die Impfung ebenso erbracht werden müsste, wie für diesen Bacillus. Dies aber ist bisher einwandsfrei nicht gelungen. Wir sind somit bisher berechtigt, diesen Bacillus als den alleinigen Erreger der Trichorrhexis nodosa anzusprechen.

Es erübrigt mir nur noch, auf die Arbeit von Hodara einzugehen, sowie auf zwei andere Publicationen, und zwar die von Essen und Barlow, die erst nach dem Congresse der deutschen dermatologischen Gesellschaft in Graz, auf welchem ich gegenüber den Ausführungen Petersens meinen hier vertretenen Standpunkt mitgetheilt habe.

Wenn ich nun die Wachsthumsverhältnisse und die sie begleitenden Erscheinungen des von mir bei der Trichorrhexis nodosabarbae gefundenen Bacillus vergleiche mit den ron Hodara angegebenen, sowie die Morphologie des des Bacillus selbst, so finde ich zwischen diesen Beobachtungen eine solche Uebereinstimmung, dass ich nicht anstehe, dieselben für identisch zu erklären und dies umsomehr, als auch mein Infectionsversuch, ebenso wie der $\mathrm{H}$ o d a ra's positiv ausfiel. Allerdings muss ich einen vielleicht nur scheinbaren durch äussere Umstände veranlassten Unterschied zwischen meinen und $\mathrm{Ho}_{\mathrm{o}}$ dara's Beobachtungen bei Stichculturen auf Gelatine hervorheben. Hodara gibt nämlich an, dass die Cultur am Ende der vierten Woche eine Spur ron Verlüssigung zeigt und sich im Laufe eines Vierteljahres auch nicht weiter verändert. Diese Angabe ist vollkommen richtig, trifft aber nur dann zu, wie 
ich mich überzeugen konnte, wenn die Gelatinecultur vor Austrocknung nicht vollkommen geschützt war.

Ich lege hierauf um so mehr Gewicht, als ein diesbezüglicher Unterschied ja die Identität dieser beiden Formen in Frage stellen würde, obwohl andererseits die von mir beobachteten morphologischen Verhältnisse vollkommen mit denen übereinstimmen, wie sie $\mathrm{H}$ o d a r a beschrieben und abgebildet hat.

v. Fssen hat sich ebenfalls mit der Aetiologie der Trichorrhexis nodos a eingehend beschäftigt. Von 23 systematisch untersuchten Haaren eines Falles entwickelten sich an 21 Colonien eines Bacillus und zwar siebenmal in Reincultur - vierzehnmal in Gesellschaft mit einem Coccus.

Herr Ur. v. Essen hatte nun die Güte, mir auf meine Bitte eine Reincultur seines Bacillus zuzusenden und die Untersuchung derselben ergab, dass sein Bacillus in der That vollkommen verschieden ist, von dem von mir gefundenen. Was nun die ätiologische Bedeutung seines Bacillus betrifft, so gelang es ihm mit Reinculturen desselben eine typische Trichorrhexis zu erzeugen; hingegen gelang es ihm nicht, wie Hodara und mir, aus dieser Impftrichorrhexis den Bacillus neuerdings zu züchten. v. Es s e n glaubt dies dadurch erklären zu können, dass die Bacillen, welche die T'richorrhexis erzeugt haben, zur Zeit der Untersuchung der Haare bereits abgestorben waren. Diese Erklärung scheint übrigens auch $\nabla$. Essen nicht ganz plausibel zu sein, denn er selbst hebt hexror, dass sich die Bacillen in den Haaren des Stammfalles sechs Monate lebensfähig erhalten haben.

Sollte v. Essen's Versuch aber volle Beweiskraft haben, so hätte er den ron ihm als Erreger der Trichorrhexis angesprochenen Bacillus wieder durch die Cultur nachweisen müssen und dieser Umstand stellt die ätiologische Bedeutung des von v. Essen gefundenen Bacillus sehr in Frage. Allerdings bleibt es unaufgeklärt - und v. Essen selbst hat dies hervorgeboben - in welcher Weise die Trichorrhexis in diesem Falle zu Stande kam. Vielleicht werden v. Essen's weitere Untersuchungen diesen Punkt aufklären.

In einer jüngst erschienenen Publication versucht $B$ arlow die positiven Ergebnisse der bakteriologischen Untersuchung 
bei Trichorrhexis nodosa einer Kritik zu unterziehen und die ätiologische Bedeutung des von Hodara, mir und v. Essen gefundenen Bacillus zu bezweifeln, wobei er ganz ungerechtfertigt die Untersuchungen von Raymond heranzieht, der bei Trichorrhexis einen Diplococcus gezüchtet hat, dessen ätiologische Beziehungen zur Trichorrhexis Raymond selbst durch nichts erwiesen hatte. Es ist daher die Behauptung Barlow's, dass bisher drei wohl unterschiedene Bakterienarten von ihren Entdeckern als Ursache der Trichorrhexis angesehen wurden, ganz unzutreffend, denn wie ich oben bereits angeführt habe, vermochte ich keinen wesentlichen Unterschied zwischen dem Bacillus von Hodara und mir ausfindig zu machen, während in Bezug auf den v. Essen's der vollgiltige ätiologische Beweis noch nicht erbracht ist, vielmehr bleibt nur eine einzige Bakterienart übrig, von welcher der Beweis vorliegt, dass sie Trichorrhexis zu erzeugen vermag.

Nachdem Barlow die mykotische Aetiologie der Trichorrhexis nodosa in Zweifel zieht, versucht er nun diese Affection durch die mechanische Theorie Wolfsberg's zu erklären.

Wenn auch zugegeben werden mag, dass durch mechanische Insulte an Barthaaren ein der Trichorrhexis nodosa ähnliches Krankheitsbild erzeugt werden kann, so hat Wolfsberg selbst am besten gezeigt, dass diese Erscheinung absolut nicht identisch ist mit Trichorrhexis nodosa, indem es ihm gelang, dieselbe durch einfaches Abschneiden der Haare zu entfernen. Dies aber lässt sich, wie wir ja wissen, bei der Trichorrhexis nodosa barbae nicht erzielen. Barlow gebt aber dieser mechanischen Theorie zu Liebe so weit, dass er es sogar ernstlich in Erwägung zieht, ob nicht die mechanischen Insulte bei der Impfung eine Rolle gespielt haben bei dem späteren Auftreten der Trichorrhexis. Nun, man wird mir wohl zugeben, dass dieses Moment am allerwenigsten in Betracht kommen kann. Bei vielen Leuten, die sich gewohnheitsmässig den Schnurrbart drehen, müsste man dann Trichorrhexis finden, was bekanntlich nicht zutrifft.

Barlow hat schliesslich an den Bartbürsten von zwei an Trichorrhexis erkrankten Personen dieselben Veränderungen constatiren können. Dies ist wohl möglich, da es sich hiebei 
um ähnliche Epidermisgebilde handelt, wie die Haare sind und könnte dieser Umstand gerade ebenso gut für die parasitäre Aetiologie als Beweis herangezogen werden, ein Argument, auf das ich als supponirt kein Gewicht lege. Bakteriologische Befunde hierüber liegen übrigens seitens Barlow's nicht vor. Was übrigens die von $\mathrm{B}$ arlow beobachtete, von ihm mit Trichorrhexis identificirte Erscheinung anbetrifft, so handelt es sich hier ja nur um mechanisches Abbrechen und Auffaserung der steifen Borsten von Bürsten, eine Beobachtung, die ich auch an Zahnbürsten sowie Bürsten jeder Art gemacht habe. Was schliesslich die negativen Ziichtungsversuche Barlow's mit Trichorrhexis-Haaren betrifft, so schwächen dieselben die positiven Ergebnisse Anderer und meiner Untersuchungen in keiner Weise ab.

Die letzte Publication über diesen Gegenstand ist die von Bruhns. Er unternahm bei sechs Fällen von Trichorrhexis Züchtungsversuche und zwar sowohl durch directe Uebertragung von Trichorrhexis-Haaren auf Nährmedien, als auch nach vorausgegangener Alkoholbehandlung. Nach der letzteren Methode machte er sechzehn Züchtungsversuche mit negativem Erfolge, bei der ersteren Methode erhielt er ein Gemisch der verschiedensten Mikroorganismen. Es ist vollkommen richtig, dass bei dieser Methode alle möglichen Mikroorganismen aufgehen und den Trichorrhexis-Bacillus möglicherweise überwuchern; was hingegen die Alkoholmethode betrifft, kann ich Bruhns nur zustimmen, dass dieselbe, wie ich schon hervorgehoben habe, nicht in jedem Falle die Züchtung der Trichorrhexis-Bacillen ernöglicht. Untersucht man hingegen mit der oben angegebenen Methode, so kann man sich von der Anwesenheit derselben überzeugen. Bruhns zweifelt ferner daran, ob der von Hodara und mir gezüchtete Bacillus uns in Reincultur vorlag und zwar in Hinsicht auf dessen Polymorphismus. Hätte aber Bruhns ebenso wie Hodara und ich denselben gezüchtet, so hätte er sich an Plattenculturen überzeugen können, dass seine Bedenken ungerechtfertigt sind. Seine Versuche, abgeschnittene gesunde Haare durch Verreiben derselben mit kranken Haaren zu inficiren, sind offenbar unzureichend, weil ja Impfversuche mit Reinculturen positiv aus- 
fallen. Wenn aber seine Impfrersuche mit Bakterien. gemisch negativ ausfielen, so kann dies daher kommen, weil dasselbe den Trichorrhexis-Bacillus nicht enthalten haben dürfte, beziehungsweise ron anderen Mikroorganismen überwuchert worden war. Ueberhaupt ist es unzulänglich, dort wo es sich um die Feststellung der ätiologischen Bedeutung eines Mikroorgan is mus handelt, mit einem Bakteriengemisch zu arbeiten.

Schliesslich sei noch eine kurze Mittheilung von Mar$\mathrm{ku}$ sfeld erwähnt, die sich gleichfalls mit der Aetiologie der Trichorrhexis nodosa beschäftigt. Markusfeld, der denselben Bacillus aus Trichorrhexis-Haaren gezuichtet hat wie ich, gelang es, meine Befunde zu bestätigen, und wenn Markusfeld sich darüber wundert, dass ich den von mir gezüchteten Bacillus mit dem Hodara's identificire, so wird ihm roraussichtlich ein genaueres Studium namentlich älterer Cultnren zu demselben Resultat führen müssen wie mich; dies bezieht sich auch auf die von ihm angegebene Sporenbildung, von deren Vorhanhensein ich mich nie überzeugen konnte.

\section{Therapentisches.}

Die Trichorrhexis nodosa barbae ist also eine parasitäre Krankheit, erzeugt durch einen dieser Krankheit eigenthümlichen Bacillus. Anatomisch ist die Trichorrhexis nodosa barbae dem Herpes tonsurans capillitii, sowie dem Favus in eine gewisse Analogie zu bringen, indem sowie bei jenen Krankheiten eine Vegetation des Krankheitserregers im Haare stattfindet und auch wie beim Herpes tonsurans die kranken Haare abbrechen. Aber auch der 'Therapie ist diese Affection mit Rücksicht auf die Verbreitung der Bakterien innerhalb der Follikel und bei der Schwierigkeit, parasiticide Salben mit dem Krankheitserreger in directe Berührung zu bringen, sehr schwer zugänglich. Durch den Umstand, dass die Bacillen in dem subepidermoidalen Theile des Haares gleichfalls vorkommen, wird die von Kaposi schon längst gemachte klinische Beobachtung, dass Rasieren nur in einzelnen Fällen Hilfe bringe, jetzt, wo wir die pathologische Grundlage dieser Krankheit erkannt haben, vollkommen erklärlich; denn indem die Krankheitserreger nach dem Rasieren in der Haar- 
wurzel zurückbleiben, wird von da aus immer wieder die Verbreitung derselben durch das Wachsthum der Haare ermöglicht. Es mag also in denjenigen Fällen, in denen durch das Rasieren Heilung erfolgte, sich die Krankheit in jenem Anfangsstadium befunden haben, in welchem die Infection des subepidermoidalen Theiles der Haare noch nicht erfolgt war, also durch das Rasieren die Krankheitserreger vollkommen eliminirt werden konnten.

Eine radicale Behandlung dieser Affection aber müsste sich auf dieselben Behandlungsprincipien stïtzen, auf welchen die des Farus und des Herpes tonsurans capillitii beruht - Ausziehen der kranken Haare, einerseits um die Krankheitserreger nach Möglichkeit auf diesem Wege zu entfernen, andererseits um die Follikel für das Eindringen parasiticider Salben zugänglich zu machen. Dies müsste mit regelmässigem Rasieren vereinigt werden. Es ist nicht wahrscheinlich, dass sich jemand dieser mit der Geringfügigkeit des Uebels in keinem Verhältnisse stehenden Unannehmlichkeit einer solchen Behandlung für längere Zeit, wie dies nothwendig wäre, unterziehen wird und dieser Vorschlag hat daher nur akademischen Werth.

Sonstige therapeutische Massnahmen können also, wie hieraus hervorgeht, das Uebel vielleicht mildern, aber nicht beheben.

\section{Epikrise.}

1. Die Trichorrhexis nodosa barbae ist eine parasitäre Krankheit, hervorgerufen durch einen mit $\mathrm{Hod}$ ara's Bacillus höchstwahrscheinlich identischen Erreger.

2. Dieser Bacillus findet sich constant in den kranken Haaren in Form von uncharakteristischen Häufchen von Coccen, als auch als bis zu $12 \mu$ grossen Stäbchen, sowohl im Haare selbst als auch mitunter im subepidermoidalen Theile von solchen Haaren, und in den Zellen der Follikelwand. Derselbe lässt sich auf den gewöhnlichen Nährbòden gut züchten.

3. Gesunde Haare zeigen weder jene Bilder-Colonien, noch auch gelingt die Züchtung jenes Bacillus aus diesen Haaren.

4. Andere Mikroorganismen vermögen, soweit bisher bekannt, nicht Trichorrhexis nodosa zu erzeugen. 
5. Da das Ziel einer rationellen Therapie in der Eliminirung des Krankheitserregers liegt, so ist neben regelmässigem Rasieren Epilation und Application von parasiticiden Salben und Epilation der Barthaare nothwendig - ein Verfahren, das allerdings nur bei sehr circumscripter Erkrankung durchführbar ist.

\section{Literatur.}

1. Abramowitsch, Beitrag zur Lehre von Trichorrhexis nodosa. Russkaja medicina 1888. refer. Archiv f. Dermatologie and Syphilis, 1889, pag. 106.

2. Anderson, Mc. C., On a unique case of hereditary trichorrhexis nodosa. Laneet 1883,2 . Bd,, pag. 140.

3. Barlow, Kurze "Bemerkungen über Trichorrhexis nodosa. Münchener klinische Wochenschrift, 1896, pag. 615.

4. Beigel, Hermann, Auftreiben und Bersten der Haare, eine eigenthümliche Erkrankung des Haarschaftes. Denkschriften der Wiener kaiserlichen Akademie der Wissenschaften 1855. Bd. XVII, pag. 612.

5. Behrend, Gustav, Ueber Knotenbildung am Haarschaft. Virchow's Archiv. Bd. 103, S. 437.

6. Idem, Verbandlungen des Berliner medicinischen Vereines. Deutsche med. Wochenschrift 1885, pag. 139.

7. Bill i, Luigi, Gionale Italiano delle Malattie Veneree. Aug. 1872.

8. B la s chko, Ueber die Hygiene der Barbierstuben. Berliner klin. Wochenschrift 1893, pag. 841 .

9. Bruhns, Zur Aetiologie der Trichorrhexis nodosa. Archiv f. Dermatologie und Syphilis. 38. Bd. 1897, pag. 43.

10. Cheadle, Lancet 1878 , pag. 313 .

11. Cheadle and Malcolm Morris, Piedra, Trichorrhexis nodosa, Tinea nodosa. Lancet 1879. I. Bd., pag. 190.

12. Desenne, Sur la "piedra“, nouvelle espèce d'affection parasitaire de cheveux, presentée par M. Vulpian. Comptes rendus T. 87, p. 34.

13. Devergi ie, Trichoptilosis. Annales de Dermatol. et Syphil. 1871.

14. Duhring, Lehrbuch der Hautkrankheiten (citirt nach T. Fox).

15. Eich orst, Beobachtungen über Trichorrhexis nodosa. Zeitschrift f. klin. Medicin. Festschrift für Frerichs. Supplement zum VII. Bd. 1884, pag. 59 .

16. Essen, O. v., Bakteriologische Untersuchung eines Falles von Trichorrhexis nodosa barbae. Archiv f. Dermatologie und Syphilis 1895 . 33. Bd.

17. T. Fox, On skin diseases. 2. Auflage, pag. 459. (Citirt nach Hoggan. Lancet 1878, pag. 347 .)

18. I d e m, Lancet 1878, pag. 420 .

19. Hodara, Ueber die Trichorrhexis des Kopfhaares der Konstantinopeler Frauen. Monatshefte f. prakt. Dermatologie. Bd. 19.

20. Hoggan, G., Lancet 1878. 2. Bd., pag. 347 .

21. Jamieson, Ein Fall von Knotenhaaren. Med. Press and Circ. London 1888. Archiv f, Derm. und Syphilis. 1890, pag. 258.

22. Kaposi, In Hebra-Kaposi. Handbuch der Hautkrankheiten. 2. Bd. 1876 , pag. 176 .

23. I d em, Pathologie und Therapie der Hartkrankheiten. 4. Auflage. 1893, pag. 700 . 
24. Kohn, S., Ueber Trichorrhexis nodosa. Vierteljahrschrift $f$. Dermatologie und Syphilis. 1881, pag. 581.

25. Malcolm A. Morris, Lancet 1878,2 . Bd.

26. Mall ey: Lancet 1878. 2. Bd., pag. 276.

27. Markusfeld, Ueber die Aetiologie der Trichorrhexis nodosa (Kaposi). Centralblatt f. Bakteriologie und Parasitenkunde. 1897.

28. Morris, M., Piedra - a new disease. Transaction of the pathological society of London. 1879, pag. 411. Citirt nach Eichhorst.

29. I d e m, Medical Times and Gazette. 1879, 12. April, pag. 409.

30. N eumann, Lehrbuch der Hautkrankheiten 1880, pag. 448.

31. Osorio, eit. nach Desenne.

32. Pye-Smith, P. H., Specimen of the affection of the hairs, wich has been described as Piedra and as Trichoclasia as Trichorrhexis nodosa. Transactions of the pathological society of London. 1879, pag. 439. (Citirt nach Eichhorst.)

33. Raymond, Recherches sur la Trichorrhexis nodosa. Annales de Dermatologie et Sypl. 1891. 1878, Nr. 3 .

34. Roeser, Trichoptilose. Annales de Dermatologie et de Syphil.

35. Schwimmer, Veber Trichorrhexis nodosa barbae. Vierteljahrschrift f. Dermatologie und Syphilis. 1878, pag. 581 .

86. S m ith, Walther G., Zwei Fälle von fragilitas crinium. August 1879. Refer. in Schmidt's Jahrbücher. Bd. 191, pag. 31 .

37. Spiegler, Trichorrhexis nodosa. Wr. med. Blätter 1895.

38. I d e m, Verhandiungen der deutschen dermatologischen Gesellschaft. V. Congress. 1895, pag. 366 .

39. Wilks, Samuel, Lectures of pathological Anatomy. 1857.

40. I de m, Lancet 1878. II. Bd., pag. 347.

41. Wh itla, Dublin Journal of med. sciences. 86. Bd. 1879, pag. 704. Refer. Vierteljahrschrift f. Dermatologie und Syphilis. 1879, pag. 401 . 42. Wils on, Erasmus, Lectures on dermatology. 1871, pag. 125. Cit. nach T. Fox.

43. W olf s berg, Zur Aetiologie der Trichorrhexis nodosa. Dentsche med. Wochensehrift, 1884, $\mathrm{Nr}$. 31 .

\section{Erklärung der Abbildungen auf Taf. $\mathrm{V}$.}

Fig. 1. Trichorrhexishaar mit Stäbchen. Ocular 3. Objectiv 2.

Fig. 2. Theil eines Trichorrhexishaares mit Stäbchen. Ocular 4. Objectiv 7 , ausgezogoner Tubus. 

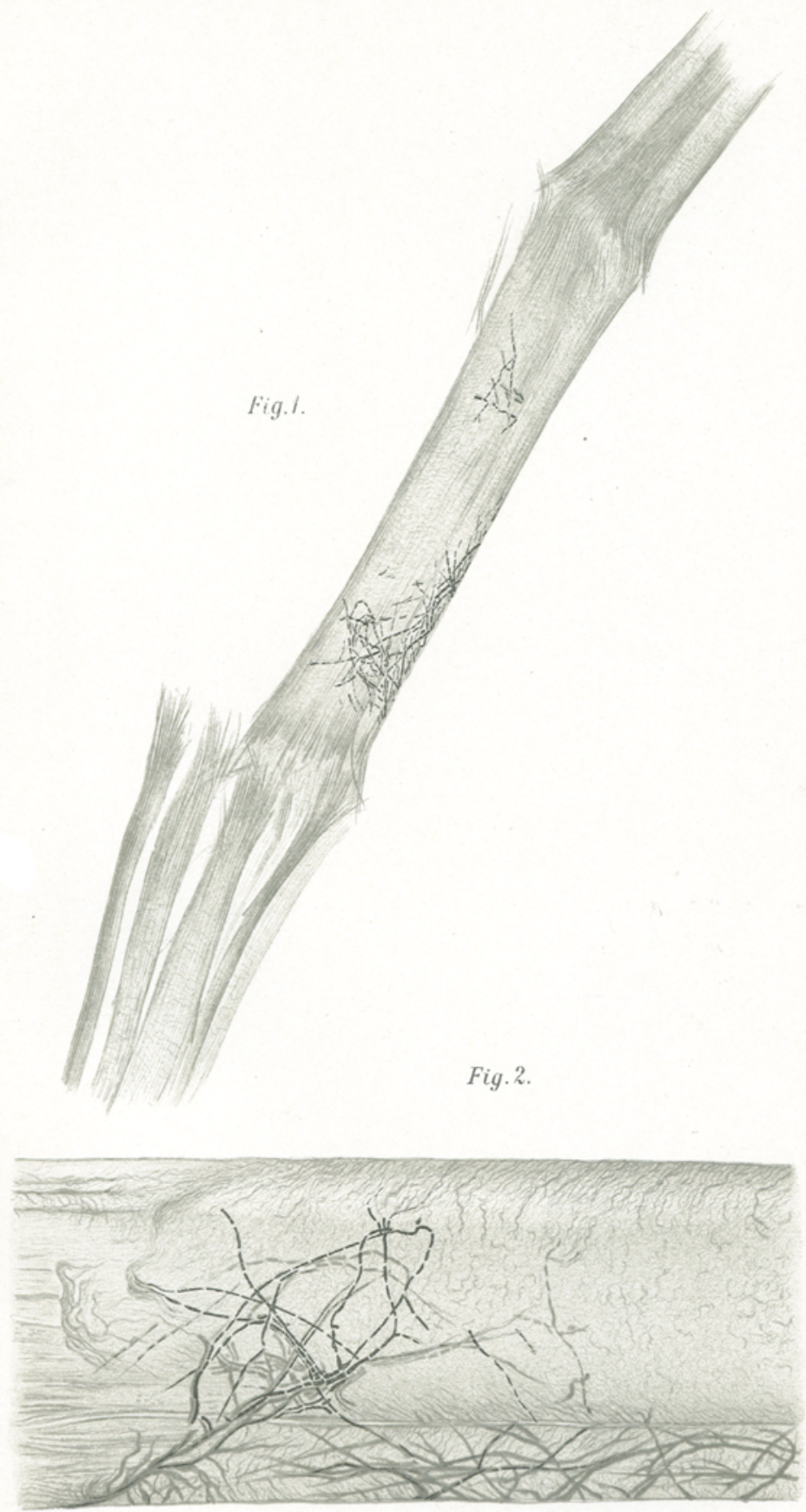

Splegler: Trichorrhexis nodosa barbae. 